\title{
Lipid Accumulation Product Index to Predict Metabolic Syndrome in People Living with HIV
}

Mariana Amaral Raposo, MSc; Nathalia Sernizon Guimarães, PhD; and Unaí Tupinambás, MD, PhD

Background: Dyslipidemia and central obesity are the main components of metabolic syndrome, which represent important risk factors for cardiovascular diseases in people living with human immunodeficiency virus (HIV). The lipid accumulation product (LAP) index has been shown in studies as an efficient marker of metabolic syndrome in general adult population and its applicability in HIVinfected population is not discussed. We aimed to assess the accuracy of the LAP index to identify metabolic syndrome in people living with HIV.

Methods: It is a cross-sectional study with I4I HIV-infected patients on antiretroviral therapy, conducted in a reference centre of infectious diseases in southeast Brazil. Evaluations included LAP index, anthropometric measurements and clinical and laboratorial variables. Metabolic syndrome was defined by International Diabetes Federation (IDF) criteria.

Results: The prevalence of metabolic syndrome in our sample was $10.6 \%$. A positive and significant correlation was found between the metabolic syndrome and LAP $(r=0.401 ; P<0.01)$, metabolic syndrome and body mass index $(r=0.361 ; P<0.01)$ and metabolic syndrome and waist circumference $(r=0.427 ; P<0.01)$ in our sample. The analysis of the receiver-operating characteristic $(R O C)$ curve revealed that the best cut-off value for LAP index to define metabolic syndrome was 59.4 (sensitivity $80 \%$, specificity $79 \%$ and area under the curve (AUC) of 0.875 . In female and male, analysis of the ROC curve revealed that the best cut-off value for LAP index to define metabolic syndrome was 56.3 (sensitivity $100 \%$, specificity $82 \%$ and AUC of 0.929 ) and 52.0 (sensitivity $78 \%$, specificity $74 \%$ and AUC of 0.838 ), respectively.

Conclusion: Despite the low prevalence of metabolic syndrome in our sample, the ROC curves analyzes demonstrated a good diagnostic accuracy as an additional screening tool of metabolic syndrome according to the IDF.

Keywords: HIV; Metabolic syndrome; Lipid accumulation product

Corresponding Author: Mariana Amaral Raposo, MSc; Av. Prof. Alfredo Balena, 190 - sala 533 / Belo Horizonte - MG, 30130-100, Brazil, Email: marianamgbh@hotmail.com

Funding Disclosure: The authors received funding for this study from Technical Cooperation DNDSTAIDS and HV-United Nations Office on Drugs and Crime Project BRA / K57 (Edital 0I/20I 3 Technical Cooperation)

Ethics Approval: The Research Ethics Committee of the Federal University of Minas Gerais approved the present study, under the protocol number 0251.0.203.000II, and all participants gave written informed consent.
Received: June 7, 2019

Revised: January I5, 2020

Accepted: March 13, 2020

doi: $10.3|2| / \mathrm{cmr} .2020 .1509$ 
$\mathrm{G}$ lobally, cardiovascular diseases represent about $30 \%$ of deaths every year and it is the leading cause of morbidity and mortality worldwide. ${ }^{1}$ Compared with the overall population, people living with HIV (PLWH) have higher cardiovascular risk. ${ }^{2}$ Shah et $\mathrm{al}^{3}$ reported, in their systematic review of longitudinal studies, that the relative risk of cardiovascular diseases in PLWH is 2.16 higher than uninfected population.

Despite the considerable increase of the survival of PLWH due to the success of antiretroviral therapy (ART), long-term ART has been associated with metabolic and cardiovascular complications as it has become an important challenge in the clinical management of HIV infection., ${ }^{4,5}$ Dyslipidemia and central obesity are the main components of metabolic syndrome (MS), which represent important risk factors for cardiovascular diseases. $^{6}$

There are several criteria for diagnoses of metabolic syndrome. The International Diabetes Federation (IDF) criteria is focused on the presence of central obesity, which is evaluated by waist circumference (WC) adapted to anthropometric profile of various populations. ${ }^{6}$

Among the anthropometric parameters to estimate body fat [Body mass index (BMI)] and measurement of adiposity (WC, waist-to-height and conicity index), the lipid accumulation product (LAP) index had been proposed as an easier and useful tool to predict lipid accumulation. ${ }^{7,8}$

Described for the first time by Kahn et al, ${ }^{7}$ the LAP index is calculated by the combination of waist circumference $(\mathrm{cm})$ and fasting triglycerides (TG) levels ( $\mathrm{mmol} / \mathrm{L}$ ). The LAP index has been shown in studies as an efficient marker of metabolic syndrome in adult populations ${ }^{8,9,10}$ and it is mainly discussed in studies with women with polycystic ovary syndrome, which metabolic abnormalities are common as well. ${ }^{11,12}$

The great variety of diagnostic methods for the detection of metabolic syndrome makes it difficult to compare the results of the studies that evaluate this comorbidity in PLWH. In view of the simplicity of execution and standardization of the measures that are part of the LAP index, this study aims to analyze the LAP index's ability to identify PLWH with metabolic syndrome according to IDF criteria after beginning of ART.

\section{Methods}

Study Design and Participants

It is a cross-sectional study with 141 PLWH in use of antiretroviral, conducted in a Reference Centre of Infectious Diseases in southeast Brazil. All the participants of this study have had medical indication for the beginning of ART.

On average, $120 \mathrm{PLWH}$ receive ART per year in this reference centre of infectious diseases. Regarding the sample calculation, we worked with metabolic syndrome prevalence of $16.7 \%$ in PLWH according to the overall prevalence found in metaanalyses of Nguyen et al. ${ }^{13}$ The margin of error of this study is $\pm 5 \%$ and $99.99 \%$ of confidence level. The minimum sample size calculated was 105 individuals.

\section{Ethical Considerations}

The Research Ethics Committee of the Federal University of Minas Gerais approved the present study, under the protocol number 0251.0.203.000-11. All participants gave written informed consent.

\section{Data Collection}

The data were collected through anthropometric, clinical and laboratorial evaluations. Height and weight were collected using standardized procedures by the World Health Organization (WHO) ${ }^{14}$ In order to measure height, the stadiometer of the anthropometric scale of the brand "Filizzolla" was used. Body weight was determined on an anthropometric scale of the Tanita brand with a capacity of $150 \mathrm{~kg}$. WC was defined as the measurement at the midpoint between the lateral iliac crest and the lowest rib. ${ }^{15} \mathrm{BMI}$ was calculated as weight $(\mathrm{kg})$ divided by height $\left(\mathrm{m}^{2}\right)$.

A modified WHO STEPS cardiovascular risk data surveillance questionnaire was administered to collect data about physical activity and lifestyle. The collected data was registered in specific forms.

\section{Assays}

Blood samples were taken after a 12-hour overnight fast. Plasma glucose, total cholesterol (TC) and TG were determined enzymatically (Vitros Chemistry Products, Johnson \& Johnson Clinical Diagnostics, Rochester, USA). High density lipoproteins (HDL) was measured using an immune inhibition assay (Labtest Diagnosis, Lagoa Santa, Brazil), and insulin was measured by chemiluminescence (Diagnostic Products Corporation, Los Angeles, CA, USA). Low density lipoproteins (LDL) was calculated. LDL was not evaluated in patients with triglyceride levels above $400 \mathrm{mg} / \mathrm{dL}$.

CD4+ T lymphocyte counts were obtained by flow cytometry (FACSCalibur Becton \& Dickinson, USA) using BD Multitest kits (Becton Dickinson, USA). Plasma viral load was determined by Real Time PCR (Abbott RealTime HIV-1 essay) (Abbott, USA).

\section{Definitions}

The IDF classifies patients with metabolic syndrome according to the presence of abdominal obesity (WC $>94 \mathrm{~cm}$ for men and $>80 \mathrm{~cm}$ for women), as a condition sine qua non, and two or more criteria, such as: TG $>150 \mathrm{mg} / \mathrm{dL} ; \mathrm{HDL}<40 \mathrm{mg} / \mathrm{dL}$ for men and $<50 \mathrm{mg} / \mathrm{dL}$ for women; systolic arterial pressure $>$ $130 \mathrm{mmHg}$ or treatment for hypertension and diastolic arterial pressure $>85 \mathrm{mmHg}$ or treatment for hypertension; and raised 
fasting plasma glucose $\geq 100 \mathrm{mg} / \mathrm{dL}(5.6 \mathrm{mmol} / \mathrm{L})$, or previously diagnosed type 2 diabetes. $^{6}$

LAP index is determined by the following equation for women: (WC $[\mathrm{cm}]-58) \times(\mathrm{TG}[\mathrm{mmol} / \mathrm{L}])$, and for men: [WC $(\mathrm{cm})-65] \times \mathrm{TG}(\mathrm{mmol} / \mathrm{L})$.

\section{Statistical Analyses}

All statistical analyses were performed using SPSS, version 20.0 for Windows (Chicago, IL, USA).

The data were described as measures of central tendency (mean or medians) and measures of dispersion [standard deviation (SD) or interquartile range (IQR)] for numerical variables. To check data normality, the Shapiro-Wilk test was applied. A variable was considered as having a normal distribution if $P<0.05$ and as asymmetrical distribution if $P>0.05$. Continuous variables were compared using the Mann-Whitney test.

The non-parametric Spearman rank correlation coefficient was used to assess agreements between metabolic syndrome and the continuous independent variables (BMI, WC and LAP index).

Variables with $P<0.1$ related to the increase in metabolic syndrome by univariate analysis were considered candidates for the logistic regression model. Stepwise backward logistic regression was used in the construction of multivariate model. Calibration and discrimination of the models were determined by the Hosmer-Lemeshow test and the receiver-operating characteristic (ROC) curve analysis, respectively.

For all tests, they were considered as level of statistical significance a $P<0.05$.

\section{Results}

The study included 141 PLWH with a median beginning of ART of 7.5 months [IQR 28] and median ART of 24 months [IQR 8]. The median age was 37 years [IQR 14]. Women were statistically older and there were no statistically significant differences between the sexes in relation to their schooling, current smoker, sedentary lifestyle, blood pressure, fasting glucose, BMI, WC, TG, TC, LAP index and metabolic syndrome. Table 1 summarizes the demographic, lifestyle, clinical and laboratory variables of the overall study sample and categorized by sex. It was possible to observe that men had higher alcohol consumption, weight, LDL and lower HDL when compared with women.

A positive Spearman's rank correlation (r) was found between the metabolic syndrome and LAP $(0.4007 ; P<0.01)$, metabolic syndrome and BMI $(0.3606 ; P<0.01)$ and metabolic syndrome and WC in our population $(0.4274$; $P<0.01)$.

Overall, the analysis of the ROC curve revealed that the best cut-off value for LAP index to define metabolic syndrome was 59.4 (sensitivity $80 \%$, specificity $79 \%$ and area under the curve (AUC) of 0.875 ). In female population, the analysis of the ROC curve revealed that the best cut-off value for LAP index to define metabolic syndrome was 56.3 (sensitivity $100 \%$, specificity $82 \%$ and AUC of 0.929$)$. In male, the ROC curve revealed the best cut-off value was 52.0 (sensitivity $78 \%$, specificity $74 \%$ and AUC of 0.838 ) (Figure 1).

\section{Discussion}

This is the first study to assess the LAP index to predict metabolic syndrome in PLWH. In Brazil, a cross-sectional

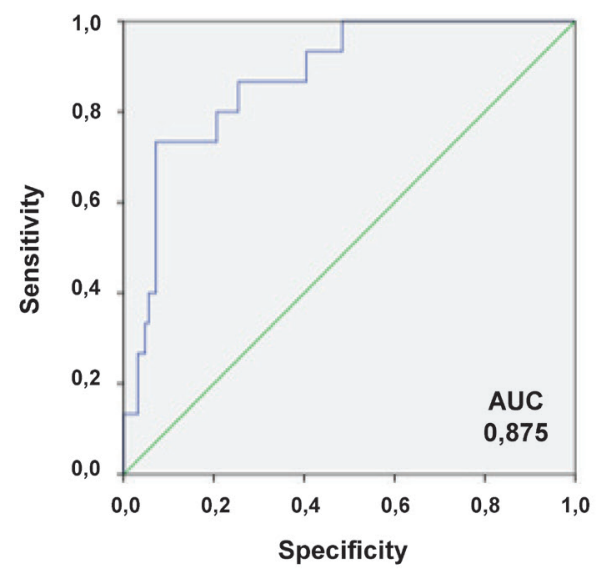

A. ROC curve for general sample

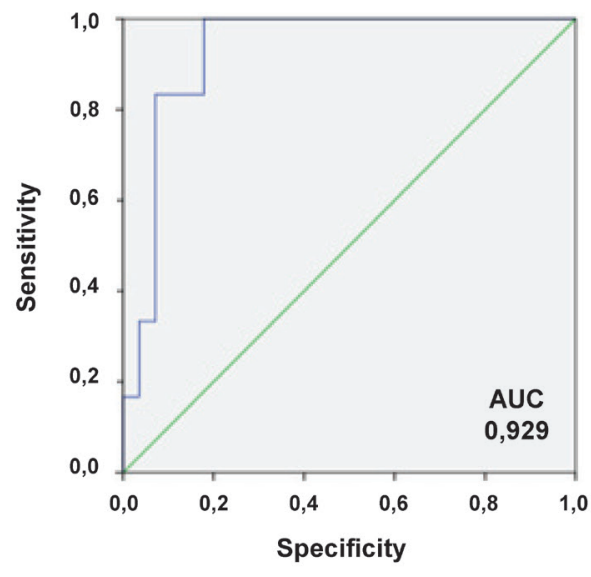

B. ROC curve for female

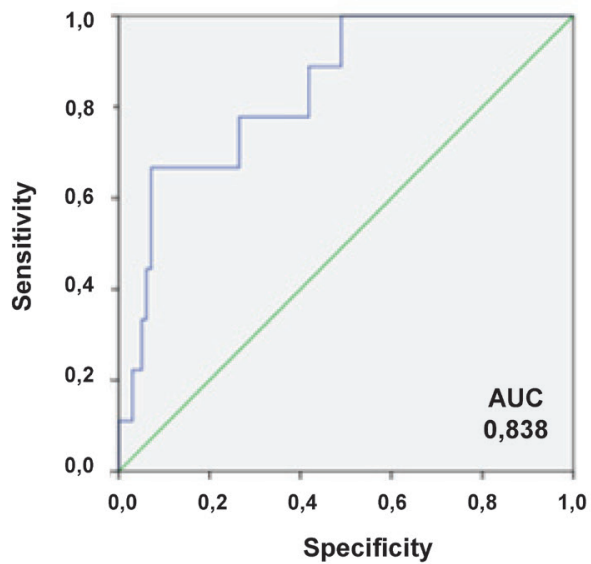

C. ROC curve for male

Figure 1. ROC curve for lipid accumulation product index as a marker of metabolic syndrome in (A) general sample, (B) females, and (C) males. 
Table 1. Clinical and metabolic features in PLWH after beginning of ART $(n=141)$.

\begin{tabular}{|c|c|c|c|c|}
\hline Variables & $\begin{array}{l}\text { Overall } \\
(n=141)\end{array}$ & $\begin{array}{l}\text { Male } \\
(n=107)\end{array}$ & $\begin{array}{l}\text { Female } \\
(n=34)\end{array}$ & $P$ value \\
\hline Age (years) & $37[14]$ & $35[14]$ & $44.5[7]$ & $<0.001^{*}$ \\
\hline \multicolumn{5}{|l|}{ Schooling } \\
\hline$<7$ years & $61(43.3 \%)$ & $43(40.2 \%)$ & $18(52.9 \%)$ & \multirow{2}{*}{$0.13^{\dagger}$} \\
\hline$\geq 7$ years & $80(56.7 \%)$ & $64(59.8 \%)$ & $16(47.1 \%)$ & \\
\hline Current smoker & $28(19.9 \%)$ & $22(20.6 \%)$ & $6(17.6 \%)$ & $0.46^{\dagger}$ \\
\hline \multicolumn{5}{|l|}{ Alcohol consumption } \\
\hline$\geq 3$ times per month & $84(59.6 \%)$ & $70(65.4 \%)$ & $14(41.2 \%)$ & \multirow{2}{*}{$0.01^{\dagger}$} \\
\hline$<3$ times per month & $57(40.4 \%)$ & $37(34.6 \%)$ & $20(58.8 \%)$ & \\
\hline Sedentary lifestyle & $40(60.6 \%)$ & $21(46.7 \%)$ & $11(50.0 \%)$ & $0.81^{\dagger}$ \\
\hline \multicolumn{5}{|l|}{ Blood pressure $(\mathrm{mmHg})$} \\
\hline Systolic & $120[20.0]$ & 120 [20.0] & $120[20.0]$ & $0.89^{*}$ \\
\hline Diastolic & $80[10.0]$ & $80[10.0]$ & $80[10.0]$ & $0.96^{*}$ \\
\hline Fasting glucose (mg/dL) & $88.0[11.0]$ & $89.0[11.0]$ & $87.0[11.0]$ & $0.38^{*}$ \\
\hline Weight (Kg) & $72.6[18.3]$ & $75.0[17.3]$ & $65.0[16.4]$ & $<0.001^{*}$ \\
\hline $\mathrm{BMI}\left(\mathrm{Kg} / \mathrm{m}^{2}\right)$ & $25.0[5.8]$ & $25.0[5.3]$ & $24.4[7.0]$ & $0.69^{*}$ \\
\hline WC (cm) & $86.0[18.0]$ & $86.0[17.5]$ & $86.0[20.4]$ & $0.94^{*}$ \\
\hline $\mathrm{TG}(\mathrm{mg} / \mathrm{dL})$ & $111.0[92.3]$ & $108.0[90.0]$ & $118.0[107.0]$ & $0.16^{*}$ \\
\hline TC (mg/dL) & $182.4( \pm 41.7)$ & $176.0( \pm 41.4)$ & $201.3( \pm 37.3)$ & $0.50^{\ddagger}$ \\
\hline LDL (mg/dL) & $131.0[5245.0]$ & $154.0[7766,3]$ & $114.0[42.0]$ & $0.007^{*}$ \\
\hline $\mathrm{HDL}(\mathrm{mg} / \mathrm{dL})$ & $44.0[15.0]$ & $42.0[12.5]$ & $54.0[25.0]$ & $0.005^{\star}$ \\
\hline LAP index & $34.4[47.3]$ & $34.1[42.2]$ & $36.1[53.2]$ & $0.70^{*}$ \\
\hline Metabolic syndrome & $15(10.6 \%)$ & $9(8.4 \%)$ & $6(17.6 \%)$ & $0.12^{\dagger}$ \\
\hline
\end{tabular}

BMI - Body mass index; HDL - High density lipoproteins; LAP index - Lipid accumulation product index; LDL - Low density lipoproteins; TC - Total Cholesterol; TG - triglycerides; WC - Waist circumference. "Mann-Whitney test; ${ }^{~}$ Chi-square Test; ${ }^{\dagger} \mathrm{T}$-test

epidemiological study conducted with 211 public employees in Belo Horizonte, the analysis of ROC curves showed that the LAP index was a significant discriminator for metabolic syndrome with sensitivity and specificity greater than $80 \%$ in the best cut-off identified in all diagnostic criteria analyzed (NCEP-ATP III updated, IDF, AHA/NHBLI and harmonized NCEP-ATP III/IDF). ${ }^{8}$

Guimarães et $\mathrm{al}^{16}$ demonstrated in a cross-sectional analysis prior to this study that the LAP index was correlated with the homeostasis model assessment - insulin resistance (HOMAIR) index, a useful surrogate index of insulin resistance, constituting an additional risk factor to cardiovascular diseases in male HIV-infected patients. Unlike our study, patients with metabolic disorders such as hyperlipidemia, diabetes mellitus and lipodystrophy prior to the diagnosis of HIV-infection were excluded. The authors suggested that the screening of LAP and HOMA-IR index as part of the clinical protocol of this population could be useful even before the beginning of the symptoms of cardiovascular diseases. ${ }^{16}$

The LAP index demonstrated a good diagnostic accuracy of metabolic syndrome in our population according to the IDF criteria, as all the AUC, overall and for both sexes, were above $0.80(85 \%)$. Our findings are corroborated by studies that evaluated the LAP index to identify metabolic syndrome in the overall population. ${ }^{8,10,17}$ Ray et $\mathrm{a}^{17}$ have shown that LAP index is a better predictor of metabolic syndrome compared to BMI and WC. Although LAP, BMI and WC were independently associated with metabolic syndrome, LAP have had the highest prediction accuracy.

In our study, the LAP index presented a weak positive correlation with metabolic syndrome as well as the other anthropometric markers evaluated. The low prevalence of metabolic syndrome in our sample may have affected the 
correlation analysis between the variables, yet the correlation between metabolic syndrome and LAP index was similar to the correlation between metabolic syndrome and BMI. BMI is the anthropometric marker most widely adopted in the clinical practice to evaluate alterations in body mass. Considering that the LAP index is based on a combination of WC and fasting TG levels, it may better reflect the combined anatomic and physiological changes associated with lipid over accumulation than the BMI.

The prevalence of metabolic syndrome in our study is lower than other similar studies in HIV-infected population on ART. ${ }^{18-20}$ The regular medical care, nutritional support, psychological counseling and support groups available at the health service may be contributed with this low prevalence of metabolic syndrome in our sample. Besides that, the median age of our sample is lower when compared with these studies. The HIV infection is a chronic condition and the aging of this population increases the risk of non-infectious diseases and development of metabolic syndrome components. ${ }^{21}$

The higher prevalence of metabolic disorders in PLWH and its relation to the development of cardiovascular diseases are described in the literature. Many studies demonstrated that higher values of BMI, waist-hip ratio, diastolic arterial pressure, TG and low HDL at the beginning of ART can be significantly associated with the development of metabolic syndrome..$^{22-24}$

This study has been shown that LAP index, as anthropometric marker for identifying individuals with metabolic syndrome, can be promising and useful in the screening of the PLWH. However, the accuracy of the LAP index must be assessed in other studies to validate its real accuracy in this population.

The low prevalence of metabolic syndrome that may have overestimated the accuracy of the LAP index to identify metabolic syndrome in our sample would be one of the limitations of this study. Our sample is representative of the population of a reference centre of infectious diseases in Southeast Brazil. Therefore, the results of this study may not be generalizable to other PLWH. In addition, the design of this study did not evaluate the validity of the LAP index for incident cases of metabolic syndrome. The cross-sectional design does not allow determining how long the individuals diagnosed with metabolic syndrome have this clinical condition and whether this is due to or preceded by ART. Therefore, we suggest that longitudinal studies can better identify the application of the LAP index as a predictor of metabolic syndrome in PLWH.

\section{Conclusion}

Despite the low prevalence of metabolic syndrome in our sample, the ROC curves analysis demonstrated a good diagnostic accuracy of the LAP index in order to predict metabolic syndrome according to the IDF criteria. These data reinforce the applicability of LAP as a tool for diagnostic screening.
The early detection of who are prone to develop metabolic syndrome will enable therapeutic interventions in order to reduce the development of cardiovascular diseases and provide a better quality of life to PLWH.

\section{Acknowledgements}

The authors are particularly grateful to Júlio César Miranda, medical student at the Federal University of Minas Gerais for his technical support in this study.

\section{References}

1. Mendis S, Puska P, Norrving B. Global atlas on cardiovascular disease prevention and control. Geneva: World Health Organization. 2011.

2. Currier J, Bartlett J, Bloom A. Epidemiology of cardiovascular disease and risk factors in HIV-infected patients. 2016.

Available at: https://www.uptodate.com/contents/epidemiologyof-cardiovascular-disease-and-risk-factors-in-hiv-infectedpatients. Accessed 20 Sep 2018

3. Shah ASV, Stelzle D, Lee KK, et al. Global burden of atherosclerotic cardiovascular disease in people living with HIV: systematic review and meta-analysis. Circulation. 2018;138(11):1100-1112.

4. Lazzaretti RK, Kuhmmer R, Sprinz E, Polanczyk CA, Ribeiro JP. Dietary intervention prevents dyslipidemia associated with highly active antiretroviral therapy in human immunodeficiency virus type 1-infected individuals: a randomized trial. J Am Coll Cardiol. 2012;59(11):979-988.

5. Kibirige D, Ssekitoleko R. Endocrine and metabolic abnormalities among HIV-infected patients: A current review. Int J STD AIDS. 2013;24(8):603-611.

6. Alberti KGMM, Zimmet P, Shaw J; IDF Epidemiology Task Force Consensus Group. The metabolic syndrome - a new worldwide definition. Lancet. 2005;366(9491):1059-1062.

7. Kahn HS. The "lipid accumulation product" performs better than the body mass index for recognizing cardiovascular risk: a population-based comparison. BMC Cardiovasc Disord. 2005;5(1):26.

8. Soares LM. Produto de acumulação lipídica: acurácia para identificação de portadores da síndrome metabólica em adultos [Thesis]. Belo Horizonte: Universidade Federal de Minas Gerais - UFMG. 2016.

9. Taverna MJ, Martínez-Larrad MT, Frechtel GD, Serrano-Ríos M. Lipid accumulation product: a powerful marker of metabolic syndrome in healthy population. Eur J Endocrinol. 2011;164(4):559-567.

10. Tellechea ML, Aranguren F, Martínez-Larrad MT, Serrano-Ríos M, Taverna MJ, Frechtel GD. Ability of lipid accumulation product to identify metabolic syndrome in healthy men from Buenos Aires. Diabetes Care. 2009;32:e85.

11. Xiang S, Hua F, Chen L, Tang Y, Jiang X, Liu Z. Lipid accumulation product is related to metabolic syndrome in women with polycystic ovary syndrome. Exp Clin Endocrinol Diabetes. 2013;121(02):115-118.

12. Nascimento JXPT, Chein MBC, de Sousa RML, Ferreira AS, Navarro PA, Brito LMO. Importance of lipid accumulation product index as a marker of CVD risk in PCOS women. Lipids Health Dis. 2015;14(1):62.

13. Nguyen KA, Peer N, Mills EJ, Kengne AP. A meta-analysis of the metabolic syndrome prevalence in the global HIV-infected population. PLoS One. 2016;11(3):e0150970. 
14. World Health Organization (WHO). Physical status: the use and interpretation of anthropometry. Report of a WHO Expert Committee. Geneva: WHO. Technical Report Series No 854. 1995.

15. Leite CC, Matsuda D, Wajchenberg BL, Cerri GG, Halpern A. Correlação da medida de espessura intra-abdominal medida pela ultra-sonografia com os fatores de risco cardiovascular. Arq Bras Endocrinol Metabol. 2000;44(1):49-56.

16. Guimarães MMM, Greco DB, Moreira AN, et al. Lipid accumulation product index in HIV-infected patients: a marker of cardiovascular risk. Braz J Infect Dis. 2018;22(3):171-176.

17. Ray L, Ravichandran K, Nanda SK. Comparison of lipid accumulation product index with body mass index and waist circumference as a predictor of metabolic syndrome in Indian population. Metab Syndr Relat Disord. 2018;16(5):240-245.

18. Samaras K, Wand H, Law M, Emery S, Cooper D, Carr A. Prevalence of metabolic syndrome in HIV-infected patients receiving highly active antiretroviral therapy using International Diabetes Foundation and Adult Treatment Panel III criteria: associations with insulin resistance, disturbed body fat compartmentalization, elevated C-reactive protein, and [corrected] hypoadiponectinemia. Diabetes Care. 2007;30(1):113-119.

19. Pongthananikorn S, Jantarathaneewat K, Somnikha P, Jaturapullarp S, Meksawan K. Prevalence and Risk Factors of Metabolic Syndrome in HIV-Infected Patients Receiving the Highly Active Antiretroviral Therapy. Topics Clin Nutr. 2018;33(1):41-49.

20. Paula AA, Falcão MCN, Pacheco AG. Metabolic syndrome in HIV-infected individuals: underlying mechanisms and epidemiological aspects. AIDS Res Ther. 2013;10(1):32.

21. Raposo MA, Armiliato GNA, Guimarães NS, Caram CA, Silveira RDS, Tupinambás U. Metabolic disorders and cardiovascular risk in people living with HIV/AIDS without the use of antiretroviral therapy. Rev Soc Bras Med Trop. 2017;50(5):598-606.

22. Wand H, Calmy A, Carey DL, et al; INITIO Trial International Coordinating Committee. Metabolic syndrome, cardiovascular disease and type 2 diabetes mellitus after initiation of antiretroviral therapy in HIV infection. AIDS. 2007;21(18):2445-2453.

23. Mondy K, Overton ET, Grubb J, et al. Metabolic syndrome in HIV-infected patients from an urban, midwestern US outpatient population. Clin Infect Dis. 2007;44(5):726-734.

24. Hansen BR, Petersen J, Haugaard SB, et al. The prevalence of metabolic syndrome in Danish patients with HIV infection: the effect of antiretroviral therapy. HIV Med. 2009;10(6):378-387.

\section{Author Affiliations}

Mariana Amaral Raposo, MSc*; Nathalia Sernizon Guimarães, $P h D^{*}$ and Unai Tupinambás, $M D, P h D^{*}$

*Department of Infectology and Tropical Medicine, Faculty of Medicine - Federal University of Minas Gerais (UFMG) - Belo Horizonte, Minas Gerais - Brazil 